\title{
Inovative Surgical Treatment for Intratubal Administration of Methotrexate
}

\author{
Aneta Tomescu \\ Cezar Laurentiu Tomescu \\ "Ovidius" University of Constanţa, Faculty of Medicine, Constanta, Romania
}

Rodica Sîrbu

"Ovidius" University of Constanţa, Faculty of Pharmacy, Constanta, Romania

\begin{abstract}
In patients where conservative medical treatment with methotrexate failed, surgical intervention was necessary. The personal surgical method was performed on a number of 9 patients; the surgical intervention was a conservative method - salpingorraphy. In order to prevent persistent gestational throphoblastic disease and tubal clogging, a polyethylene catheter is introduced in the oviduct until it reaches the ostium of the Fallopian tube, without exceeding it. In order to ensure a rigorous haemostasis, the Fallopian tube and the polyethylene catheter are sutured together. The level of HCG is measured in the fourth and seventh day postoperative: if the levels of HCG are higher than $1000 \mathrm{mUl} / \mathrm{ml}$, if they plateau or if they have increased, methotrexate is administered intraluminally through the polyethylene tube, according to the following scheme: $50 \mathrm{mg}$ of methotrexate are dissolved in $10 \mathrm{ml}$ of physiological saline solution and injected through the polyethylene catheter, for 5 days. $10 \mathrm{mg}$ per day in 2 doses ( $5 \mathrm{mg}$ in the morning and $5 \mathrm{mg}$ in the evening).If the values of HCG decrease to an adequate level and there is no risk of persistent throphoblastic disease, the polyethylene catheter is kept for 8-12 days, afterwards it is extracted by traction. If the values do not decrease accordingly, the intraluminal administration of methotrexate is resumed after a 3-day break. This method was used on a number of 9 patients; 4 of them had been treated with methotrexate before the surgical intervention and pertain to the study, and 5 of them had been operated on for complications of ectopic pregnancy and were suitable for this kind of surgical intervention. No postoperative complications or deaths were recorded.
\end{abstract}

Keywords: ectopic pregnancies, methotrexate, Human Chorionic Gonadotrophin Hormone.

\section{Introduction}

Regardless of the approach used, the patient must be followed by measuring the values of HCG, because of the persistent trophoblastic disease which continues to secrete placentary chorionic hormone and also continues to increase the rate of failure for methotrexate treatment.

Methotrexate can be used in the treatment of ectopic pregnancy in the following ways: systemic, in single dose of $50 \mathrm{mg} / \mathrm{m}^{2}$ of body surface area or multiple dose: $50 \mathrm{mg} /$ day in 4 doses, $1 \mathrm{mg} / \mathrm{kg}$ body $/$ day; it can be injected directly in the amniotic sac, using ultrasound or laparoscopy. All of these methods stop the evolution of extrauterine pregnancy.

Persistent gestational trophoblastic disease is not associated only with systemic treatment with methotrexate, but also with conservative surgical interventions performed through classic or laparoscopic methods. The patient must be followed by measuring HCG 7 days postoperative; if values of HCG are greater than $1000 \mathrm{mUI} / \mathrm{ml}$ or stay at a constant level, it is necessary to administer systemic methotrexate according to the known protocol schemes and to measure the level of HCG weekly until it lowers to under $10 \mathrm{mUl} / \mathrm{ml}$.

In certain situations (haemodynamic instability and/or haemoperitoneum due to tubal rupture), surgical intervention is necessary. If a conservative surgical procedure is elected - tubal milking, salpingectomy, salpingostomy - there is the risk of secondary bleeding due to the weak muscles of the oviduct or because of the remaining trophoblastic tissues (persistent trophoblastic disease) [1,2]. The surgical technique (conservative or radical) concerning the affected oviduct will be decided intraoperatively, according to the lesions found and to the patient's wish to have children or not [3]. Under these 
circumstances, we have conceived an intraoperative surgical technique meant to solve the persistent trophoblastic disease through the intratubal administration of methotrexate using a polyethylene catheter. The polyethylene catheter, which is inserted in the oviduct, is used to inject methotrexate, guided by the values of HCG.

Late results are monitored by performing hysterosalpingography or contrast ultrasound at least 3 months after the surgical procedure, when the permeability of the ipsi- and contralateral tube is checked.

Tubal surgery and conservative surgery in general require a lot of patience and tenderness in gestures, a specialized surgeon, fine instruments and suture wires, atraumatic needles, a catheter of polyethylene. The best method in tubal plastic surgery is the one which least damages the mucosal lining [4].

\section{Research Methods}

\section{Selecting the Cases}

The patients were treated with methotrexate for ectopic pregnancy. Methotrexate therapy failed and surgery was required because of haemodynamic instability and complications.

The procedure can be used in patients not previously treated with methotrexate, in which disease became complicated, making the surgical intervention necessary.

\section{Preoperative Preparation}

Usually, surgery is a medical-surgical emergency, so there is no time for adequate preparation 2-4 before operation.

It is necessary and mandatory to get patient approval and a signed informed consent. The surgeon should warn the patient about the surgical intervention and about the therapeutic possibilities, according to the situation of the intraoperative lesions. The patient must know that the doctor will try to choose a conservative surgical procedure that will protect the pregnant tube, but the final decision will be made strictly intraoperatively, based on the lesions found in the internal genitelia. The situation becomes even more dramatic when the patient already has an ectopic pregnancy operated by radical procedure (salpingectomy).

Her obstetrical future depends on this new surgery and the risk of losing the only remaining tube is huge.

The patient must be aware and informed of the possibility of losing her only Fallopian tube, which will lead to her enrolment on a waiting list for in vitro fertilization.

In the fortunate event when the oviduct can be preserved, she must understand that conservative surgical procedures are based on strict anatomical principles, but there still remain unresolved physiological factors: muscular, epithelial, ciliary, hormonal, sperm migration, fertilization, migration of the zygote. The fact that the patient already has an ectopic pregnancy is the result of tubal morphophysiological disorders of the local and general aetiopathogenic factors, which surgery cannot solve.

The patient must know clearly before surgery all the aspects mentioned above and agree by signing an informed consent.

\section{Anaesthesia}

The type of anaesthesia, as well as the anaesthetic risk is determined by the anaesthetist. If the patient develops haemodynamic instability, the anaesthesia will be performed via orotracheal intubation. If the patient haemodynamically balanced, with normal blood pressure and pulse, without signs of haematological decompensation, a spinal anaesthesia or a peridural anaesthesia can be performed.

- Operative technique - surgical steps:

- Opening the abdominal wall;

- Inventory of lesions;

- Choice of surgical procedure

\section{Opening the abdominal wall}

Laparatomy using the Pfannenstiel incision is recommended for is aesthetic character and also because it provides an accessible way to the pelvic organs and abdominal wall closure has a low risk of eventration. 
An autostatic retractor is positioned on the abdominal wall, the intestinal loops are isolated with soft sterile fields, the patient is placed in Trendelenburg position and, afterwards, the inventory of lesions is begun.

\section{Inventory of the lesions}

The condition of the uterus, the contralateral tube, the ovaries and the presence or absence of haemoperitoneum is checked, after which, the pregnant tube is investigated. In order to reveal the oviduct, a resorbable thread is applied on the bottom of the uterus, on the median line, which is tractioned using a Pean clamp. A second resorbable wire, handled with a traction clamp, is passed at the inferior pole of the ovary. If both wires are pulled, the tube's entire trajectory is revealed. The tube is checked from the fimbria to its insertion on the uterus. The following situations can be encountered, and these also represent the indications for the personal procedure:

- Tube is pregnant but intact;

- $\quad$ Tube is intact but the embryo is free in the peritoneal cavity (tubal abortion);

- $\quad$ Tube with tubal abortion but with little continuity solution without major bleeding;

- $\quad$ Tube pregnant in the ampullar/fimbrial region and/or isthmic region, unruptured;

- Tube pregnant in the ampullar/fimbrial region and/or isthmic region, ruptured, but with minor parietal damage and without heavy bleeding.

\section{Contraindications of the personal procedure}

- Tube pregnant in the ampullar/fimbrial region and/or isthmic region, ruptured, but with important mural destruction and heavy bleeding with haemodynamic instability - radical procedure is used - salpingectomy.

\section{Results and Discussions}

\section{Electing the procedure}

Description: A polyethylene catheter is introduced into the lumen of the tube, up to the level of the uterine ostium, without exceeding it. If the tube has minor lesions or if a linear salpingectomy was performed on the antimesometrial border of the oviduct, with the purpose of evacuating the pregnancy, a salpingorraphy with nonresorbable fine 4.0 wire is performed. In order to ensure a rigorous haemostasis, the Fallopian tube and the polyethylene catheter are sutured together. The exterior diameter of the polyethylene catheter must be between 1.2-1.7 mm, because thicker catheters lead to the atrophy of the tubal mucosa through compression [4].

After ensuring haemostasis through salpingorraphy, the polyethylene catheter is kept in the oviduct; towards the uterine ostium, it is put in place with a thin, resorbable, 4.0 wire, towards the abdominal ostium, the plastic tube is attached to the serosa of the fimbria with the same kind of wire.

The permeability of the polyethylene catheter is checked by introducing either a stylet or sterile substances (physiological saline solution, sterile methylene blue). The abdominal end of the tube is exteriorized to the abdominal wall in the right or left iliac fossa, through a contraincision. An abdominal lavage with warm physiological saline solution is performed. In order to avoid the forming of tubal adhesions, a solution of dissolved dexamethazone in $10 \mathrm{ml}$ of physiological saline solution can be introduced in the polyethylene catheter. After cleaning the abdominal cavity and ensuring haemostasis, the abdominal wall is closed, layer by layer.

\section{Postoperative care:}

- $\quad$ Antibiotherapy 5 days;

- $\quad$ Prophylaxis of thromboembolic disease by administration of heparins with low molecular weight (Clexane 40$60 \mathrm{mg} /$ day depending on patient weight) and early mobilization;

- Monitoring of urine output;

- $\quad$ Stimulation and the resumption of intestinal transit;

- Determination of HCG on the fourth and seventh day after surgery - if HCG levels are higher than $1000 \mathrm{mIU} / \mathrm{ml}$, have remained in plateau or have increased, intraluminal methotrexate is administered in the polyethylene catheter, according to the treatment scheme:

- Methotrexate is administered daily through the polyethylene catheter for 5 days, in 2 doses ( $5 \mathrm{mg}$ in the morning and $5 \mathrm{mg}$ in the evening). 
- $\quad$ The polyethylene catheter is kept 8-12 days after which it is extracted by simple traction, if the HCG levels drop there is no risk of persistent trophoblastic disease.

- If the values of HCG decrease to an adequate level and there is no risk of persistent trophoblastic disease, the polyethylene catheter is kept for 8-12 days, afterwards it is extracted by traction.

If the values do not decrease adequately, the intraluminal administration of methotrexate is resumed after a 3-day break. We administer methotrexate through the polyethylene catheter inserted in the oviduct, in order to avoid the adverse effects of its systemic administration. The methotrexate administered intraluminally is diluted and it is given in very small doses in order not to damage the tubal mucosa.

Table 1 - Late results after the surgical intervention through personal procedure.

\begin{tabular}{|c|c|c|c|c|c|}
\hline \multirow{3}{*}{ 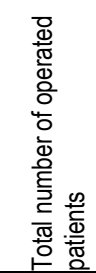 } & \multirow{3}{*}{ 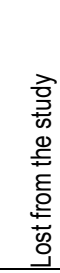 } & \multicolumn{4}{|c|}{ Pregnancies } \\
\hline & & \multicolumn{3}{|c|}{ Intrauterine pregnancies } & \multirow{2}{*}{ Extrauterine } \\
\hline & & Full term & Premature & Abortion & \\
\hline 9 & 2 & 2 & 1 & 1 & 2 \\
\hline
\end{tabular}

The general adverse reactions, decreased haemoglobin, platelets, leucocytes, the increase of transaminase, urea, creatinine, uric acid were insignificant.

If methotrexate is not required because the HCG levels drop satisfactory after surgical intervention (under $1000 \mathrm{mUI} / \mathrm{ml}$ ), a solution containing antibiotics and cortisone can be given through the polyethylene catheter, 6 days after surgery, in order to avoid the forming of adhesions. $1 \mathrm{~g}$ of ceftriaxone (cephalosporin) and $8 \mathrm{mg}$ of dexamethasone are dissolved in $10 \mathrm{ml}$ of saline solution, after we have made sure the patient is not allergic. The solution is injected through the polyethylene catheter, after which we clamp the catheter to ensure that the instilled substances come into contact with the wall of the oviduct. Consecutively, the polyethylene catheter is unclogged.

The procedure can be repeated every 3 days because the cortisone may impede tubal scarring.

The procedure was used on a number of 9 patients, 4 of them had been treated with methotrexate before the surgical intervention and they pertain to the study, and 5 of them were operated for complications of ectopic pregnancy and were suitable for this type of surgical intervention.

No postoperative complications or death were recorded.

It is premature to draw conclusion after so few interventions of this kind, but the results have been encouraging. Of the 9 patients 2 were lost from the study. Tubal patency was tested on the remaining 7 patients by performing hysterosalpingography 3 to 6 months after surgery. Tubal patency was positive in 4 patients for tubes operated through the surgical procedure. The results of the operations are estimated by tubal pregnancy and not by obtaining tubal patency [4]. Out of the 7 patients, 5 remained pregnant with intrauterine pregnancies, of which: 2 delivered full term healthy babies, one had a premature delivery (34 weeks - foetus $2100 \mathrm{~g}$ ) and 2 patients had a spontaneous abortion in the first trimester.

Two patients had a recurrent extrauterine pregnancy.

After intraluminal administrations of methotrexate, the values of HCG dropped under $1000 \mathrm{mU} / \mathrm{ml}$ after 12 days from the operation, and the values came back to normal in 23 to 31 days after the surgery. The decrease of the HCG level after conservative treatment takes more time than after the surgical intervention $[5,6]$. The procedure is similar to plastic tubal operations which ensure tubotubal anastomosis and/or tubo-uterine reimplantation surgeries. 
Technical results proved in time will probably be higher than those offered by plastic tubing operations. According to Palmer, quoted by Sarbu, the results of tubal plastic surgery are:

- More than 40\% success recorded in salpingolysis (55-75\%) and tubal reanastomosis (40-66\%);

- $\quad$ Around $30 \%$ successful in:

- Tubal reimplantation (27-38\%);

- $\quad$ Lysis of adhesion (29-35\%);

- Terminal neosapingostomy (26-32\%).

Failures, even when the operation has managed to obtain patent tubes, shows that the mere restoration of permeability only partially resolves the functional disorders of the oviduct.

We have to compare this surgical procedure which uses a classic laparatomy to surgical laparoscopy which has taken a great momentum after 1980.The success rate for laparoscopic treatment varies with different studies: the success rate of treatment $88.1 \%$, the conceiving rate post laparoscopic intervention $77.3 \%$, and the rate for recurrent pregnancy $10.6 \%$ [1]. Out of the surgical procedures used in laparoscopy, we mention: salpingectomy, salpingotomy, salpingostomy, fimbrial aspiration, peritoneal fluid aspiration and lavage, lysis of ovarian and peritubal adhesions, partial ovary resection, hysterotomy, surgery of the contralateral oviduct [1].

The complications for conservative laparoscopic surgery are significantly higher than those of radical laparoscopic surgery. The rate of complications for laparoscopic surgery is higher than that for classic laparotomy [7].

After laparoscopic salpingotomy, persistent trophoblastic disease has a higher incidence, comparatively with classic salpingotomy [8].

\section{Conclusion}

- The rate for complications after laparoscopic salpingotomy is higher than the failure rate after salpingotomy performed via a laparotomy (15.5\% as opposed to $1.8 \%)$ [7].

- In emergency cases, laparotomy remains the surgical procedure available to all obstetrical-gynaecology doctors.

- The attitude and mentality of experienced doctors has to be changed in the direction of conservative tubal surgery.

- This personal method of administration of methotrexate may protect a fragile and sometimes unique tube; it avoids the adverse effects of systemi administration and increases hopes for the patient's obstetrical future.

- If the HCG values do not require intratubal administration of methotrexate, this device can be used to prevent the forming of tubal adhesions by intraluminal administration of antibiotics and cortisone substances.

- The number of cases in which the personal procedure was used is relatively small, but the results obtained are encouraging and close to those of laparoscopic surgery.

\section{References}

[1] ANCAR V., IONESCU C. Obstetrica. Editura National ISBN 973-659-094-1, pp 223

[2] BANCEANU G. Sarcina ectopica Ministerul Sanatatii Ghid terapeutic pentru unele urgente obstetricale. Editura medicala 1988, pp 145

[3] CLASEN, K., CAMUS M., TOURNAYE H., AND DEVROEY P. - Ectopic Pregnancy; let's cut. Strict laparoscopic approach to 194 consecutive cases and review of literature on alternatives. Human Reproduction 1997, vol. 12, no. 3, pp 596-601.

[4] FERNANDEZ H, PAUTHIER, S, DOURMEC, S.1995. Ultrasound-guided injection of methotrexate (MTX) versus laparoscopic salpingotomy in ectopic pregnancy. FertilSteril, no 63, pp 25-29.

[5] HAJENIUS P, MOL BW., BOSSUYT PM., ANKUM WM., AND VAN DER VEEN. Interventions for tubal ectopic pregnancy. 2002. (Cochrane Review). The Cochrane Library

[6] HAJENIUS PJ. - Interventions for tubal ectopic pregnancy. Posted 24.01.2007 in http://www.ncbi.nlm.nih.gov/pubmed/17253448?.ordinalpos=1\&itool=EntrezSystem2.PEntrez.Pubmed/Pubmed ResultsPanel.Pubmed_DefaultReportPanel.Pubmed_RVDocSum. 
[7] LANDSTROM G., THORBURN J. AND BRYMAN I. - Treatment failures and complications of ectopic pregnancy; changes over a 20 year period. Human Reprod vol 13, no 1, 203-207-1998.

[8] SIRBU P. ARISTIDE P., CHIRICUTA, I, SETLACEC D. Chirurgica ginecologica,vol. 1, pp 212 